Ágora Rev. Cient. 2017; 04(02):e3

\title{
Factores que influyen en el nivel de cumplimiento de la lista de chequeo de cirugía segura en el Hospital San Juan de Lurigancho, 2017
}

\author{
Factors that influence on the level of compliance of the surgical safety checklist in the Surgical Center \\ in San Juan de Lurigancho Hospital, 2017
}

Ruth Condor Rios ${ }^{1 \mathrm{a}}$, Aleida Vallejos Culqui ${ }^{1 \mathrm{a}}$, Victor Humberto Chero Pacheco ${ }^{1 \mathrm{~b}}$

\section{RESUMEN}

Objetivo: Determinar los factores que influyen en el nivel de cumplimiento de la calidad de registro de lista de chequeo de cirugía segura en el centro quirúrgico del Hospital San Juan de Lurigancho, 2017. Materiales y Métodos: El estudio fue descriptivo y analítico. Con diseño no experimental y de corte transversal. La muestra estuvo conformada por 20 enfermeras del centro quirúrgico del Hospital de San Juan de Lurigancho. Se elaboraron dos instrumentos, para los factores y para el nivel de cumplimiento siendo considerada la escala de Likert y escala dicotómica respectivamente. Resultados: Se realizó el análisis estadístico, dentro de los hallazgos se encontró que los factores que influyen en el nivel de cumplimiento de la calidad de registro de lista de chequeo de cirugía segura en el centro quirúrgico del hospital San Juan de Lurigancho fueron el factor administrativo y tecnológico rechazándose la hipótesis propuesta. Conclusión: El factor administrativo y el factor tecnológico influyen significativamente en el nivel de cumplimiento de registro de lista de chequeo de cirugía segura.

Palabras clave: Factores contributivos, registro en la lista de chequeo de cirugía segura.

\begin{abstract}
Objective: To determine the factors that Influence on the Level of Compliance of the surgical safety checklist in the Surgical Center in San Juan de Lurigancho Hospital, 2017. Materials and Methods: The research was descriptive and analytical. The design was non-experimental and cross-sectional. The sample included 20 nurses in the Surgical Center in San Juan de Lurigancho Hospital. Two instruments were developed according to the factors and according to the level of compliance. A Likert scale and a dichotomous scale respectively. Results: Statistical analysis was performed, within the findings, it was found that the factors that influence on the level of compliance of the surgical safety checklist in the Surgical Center in San Juan de Lurigancho Hospital were the administrative and technological factors, so rejecting the proposed hypothesis. Conclusion: The administrative and technological factors influence in a significant way on the level of compliance of the surgical safety checklist in the Surgical Center in San Juan de Lurigancho Hospital.
\end{abstract}

Keywords: contributory factors, registration in the surgical safety checklist.

${ }^{1}$ Universidad María Auxiliadora. Lima - Perú.

${ }^{a}$ Autor

${ }^{\mathrm{b}} \mathrm{Co}$-autor

\section{INTRODUCCIÓN}

La lista de chequeo de cirugía segura es una guía que permite recordar los puntos que deben ser inspeccionados en función de los conocimientos que se tienen sobre las características y riesgos de cirugía. Es importante porque permite el desarrollo de evaluaciones que involucran complicaciones en centros hospitalarios y las características respecto al cumplimiento de las normas básicas de atención. La lista de chequeo se fundamenta en tres principios: Primero, la simplicidad, ya que es una lista en donde sólo se tocan los puntos clave y de fácil aplicación; segundo, la Amplitud de aplicación, es decir, se logra la aplicación en cualquier procedimiento quirúrgico y en cualquier nivel de equipamiento y de recursos humanos $y$, el tercero, la mensurabilidad, esta nos permite medir el impacto (1). 
Respecto a la iniciativa para la creación de una lista de control, la Organización Mundial de la Salud (2) lanzó, en el año 2008, el "Segundo reto mundial para la seguridad del paciente". Dicha iniciativa propuso la implementación de una lista de verificación de seguridad, con el objetivo de garantizar que se cumplan las normas imprescindibles de seguridad en los procedimientos quirúrgicos.

Considerando además, que los procedimientos quirúrgicos señalados en el párrafo anterior se hallan relacionados a complicaciones por no considerar un adecuado control; el Ministerio de Salud (3) señala que, tales complicaciones están asociadas con la identificación incorrecta de la zona operatoria, alergias en pacientes, complicaciones anestésicas y altos índices de infección en la herida operatoria, las mismas que se consideran de responsabilidad del equipo de salud; esto, contraviene a los principios de cirugía segura que en la actualidad se viene implementando a nivel nacional.

Para tratar de controlar las complicaciones señaladas, se toman en cuenta factores en relación al cumplimiento de actividades durante los procedimientos quirúrgicos o lista de chequeo; tenemos así, el factor humano, conformado por el personal de salud el cual puede estar ausente o afectado en su estado de salud, no pudiendo adherirse adecuadamente a los protocolos, lo cual sería desfavorable. Otro factor a considerar es el administrativo, que está conformado por la organización y gerencia. La organización del hospital; implica programar capacitaciones al personal, diseño de programas de identificación, reportes, seguimiento, análisis, plan de mejoramiento y retroalimentación de eventos adversos, garantizando la confidencialidad, programación de tiempos quirúrgicos, que incluyan tiempos para aplicación de listas de chequeo. La gerencia es la que está encargada de políticas, recursos, carga de trabajo; si la toma de decisiones no es la adecuada puede contribuir al error. Un tercer factor es el físico, que está conformado propiamente por el lugar o ambiente ya sea hospital o clínica, lo que puede contribuir al error por deficiencias; por ejemplo, en infraestructura inadecuada por falta de energía eléctrica. Finalmente, el factor tecnológico, que está conformado por los instrumentos, aparatos y equipos que al no encontrarse en buena condición pueden contribuir al error (4).

Habiendo identificado los factores asociados al cumplimiento de la lista de chequeo, cabe resaltar la incidencia de eventos adversos que ponen en peligro la calidad de atención en los servicios de salud. Estadísticamente, más de 100 millones de personas requieren tratamiento quirúrgico cada año, de los cuales en el $25 \%$ de los casos pueden presentarse complicaciones; de éstos, 7 millones son incapacitantes y entre el 0.5 y $5 \%$ causan la muerte después de la cirugía. El error técnico es el más común y el $70 \%$ de los eventos adversos se considera prevenible (5).

Señalando algunas características respecto a los eventos adversos, en el año 2009, se ha identificado una prevalencia global de eventos del $10.5 \%$; en donde los pacientes con mayor riesgo son aquellos sometidos a procedimientos quirúrgicos y los de obstetricia, en los cuales sobresale la presencia de infecciones de herida quirúrgica en $8.2 \%, \quad \mathrm{y}$ complicaciones relacionadas con intervenciones quirúrgicas $\mathrm{o}$ procedimientos con $6.4 \%$ (6).

Estudios en instituciones de salud concuerdan al identificar factores asociados a los eventos adversos mencionados. Se ha determinado, por ejemplo, que el número de intervenciones quirúrgicas representa más de 125.000 cirugías anuales, las complicaciones atribuibles a intervenciones quirúrgicas causan discapacidad o prolongan la hospitalización de los pacientes, dependiendo de la complejidad de la operación y del entorno hospitalario. Existe falta de acceso a una atención quirúrgica de calidad, por ausencia o deficiencia de habilidades y competencias, no adherencia por el personal de salud al no respetar procedimientos y protocolos, comunicación ausente o deficiente entre el equipo de trabajo, inadecuadas políticas, mal uso de recursos, exagerada carga de trabajo, deficiencias en infraestructura entro otros (7).

\section{MATERIALES Y MÉTODOS}

El estudio realizado considera el carácter descriptivo y analítico, además de ser un estudio no experimental y transversal. La población estuvo conformada por las enfermeras del hospital San Juan de Lurigancho. La muestra estuvo constituida por 20 enfermeras de sala de operaciones (SOP) del hospital San Juan de Lurigancho. Se consideró el muestreo no probabilístico. La información respecto a las variables: Factores influyentes y Nivel de cumplimiento de la lista de chequeo, fue registrada por medio de instrumentos elaborados con base en la escala de Likert y escala dicotómica respectivamente. El instrumento correspondiente a los factores, fue adaptado a partir del protocolo de Londres (8) y estuvo conformado por 15 ítems cuya medición consideró la escala ordinal. El instrumento correspondiente al nivel de cumplimiento de la lista de chequeo fue elaborado 
según la lista de chequeo creada por la OMS (2) y estuvo conformado por 20 ítems cuya medición consideró la escala dicotómica. Los instrumentos fueron validados mediante juicio de expertos y la confiabilidad fue establecida por medio de la aplicación del instrumento con una muestra piloto y la prueba Alfa de Cronbach. El procesamiento de datos fue desarrollado por medio del programa estadístico SPSS en su versión 24, considerando el análisis estadístico descriptivo, test de normalidad de Shapiro-Wilk, prueba exacta de Fisher y la prueba Chi cuadrado.

\section{RESULTADOS}

Respecto al factor humano y el nivel de cumplimiento de registro de lista de chequeo de cirugía segura. Hay más del $20 \%$ de casillas con frecuencias esperadas menores de 5 , por tanto no se puede usar prueba Chi cuadrado. Se usa la Prueba exacta de Fisher dado que la tabla de contingencia es de $2 \times 2$ y hay dos celdas con frecuencias menores de 5. Si p-value (sig.) $<$ Nivel de significación se rechaza $\mathrm{H} 0$, en caso contrario no se rechaza H0. 1.000 no es menor de 0.05 , entonces no se rechaza $\mathrm{H} 0$, por tanto con $5 \%$ de nivel de significación El factor humano y el nivel de cumplimiento de registro de lista de chequeo de cirugía segura, son independientes (Tabla 1).

Tabla 1. Factor humano y nivel de cumplimiento de registro de lista de chequeo de cirugía segura en el centro quirúrgico del hospital San Juan de Lurigancho, 2017.

\begin{tabular}{|c|c|c|c|c|c|}
\hline & Valor & Gl & $\begin{array}{c}\text { Sig. } \\
\text { asinticatica } \\
\text { (2 caras) }\end{array}$ & $\begin{array}{c}\text { Significación } \\
\text { exacta } \\
\text { (2 caras) }\end{array}$ & $\begin{array}{c}\text { Significación } \\
\text { exacta } \\
\text { (1 cara) }\end{array}$ \\
\hline Chi-cuadrado de Pearson & $.220^{\circ}$ & 1 & ,639 & & \\
\hline Corrección de continuidad & ,000 & 1 & 1,000 & & \\
\hline Razón de verosimilitud & .229 & 1 & ,632 & & \\
\hline Prueba exacta de Fisher & & & & 1,000 & ,561 \\
\hline Asociación lincal por lineal & ,209 & 1 & ,648 & & \\
\hline N de casos valilidos & 20 & & & & \\
\hline
\end{tabular}

Respecto al factor administrativo y el nivel de cumplimiento de registro de lista de chequeo de cirugía segura. Hay más del $20 \%$ de casillas con frecuencias esperadas menores de 5 , por tanto, no se puede usar prueba Chi cuadrado. Se usa la Prueba exacta de Fisher dado que la tabla de contingencia es de $2 \times 2$ y hay dos celdas con frecuencias menores de 5. Si p-value (sig.) $<$ Nivel de significación se rechaza $\mathrm{H} 0$, en caso contrario no se rechaza H0. 0,007 es menor de 0.05 , entonces se rechaza $\mathrm{H} 0$, por tanto, con $5 \%$ de nivel de significación El factor administrativo y el nivel de cumplimiento de la calidad de registro de lista de chequeo de cirugía segura, no son independientes (Tabla 2).
Tabla 2. Factor administrativo y nivel de cumplimiento de registro de lista de chequeo de cirugía segura en el centro quirúrgico del hospital San Juan de Lurigancho, 2017.

\begin{tabular}{|c|c|c|c|c|c|}
\hline & Valor & Gl & 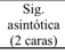 & 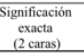 & 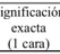 \\
\hline Chi-cuadrado de Pearson & $8,802^{2}$ & 1 & .003 & & \\
\hline Corrección de continuitad" & 6,028 & 1 & .014 & & \\
\hline Razón de verosimilitud & 9,008 & 1 & ,003 & & \\
\hline Prucba exacta de Fisher & & & & ,007 & .077 \\
\hline Assciación lineal por Iineal & 8,362 & 1 & ,044 & & \\
\hline $\mathrm{N}$ de casos válidos & 20 & & & & \\
\hline
\end{tabular}

Respecto al factor físico y el nivel de cumplimiento de registro de lista de chequeo de cirugía segura. Si p-value (sig.) < Nivel de significación se rechaza $\mathrm{H} 0$, en caso contrario no se rechaza H0. 0,372 es menor de 0.05 , entonces no se rechaza $\mathrm{H} 0$, por tanto, con $5 \%$ de nivel de significación El factor físico y el nivel de cumplimiento de la calidad de registro de lista de chequeo de cirugía segura, son independientes. (Tabla 3).

Tabla 3. Factor físico y nivel de cumplimiento de registro de lista de chequeo de cirugía segura en el centro quirúrgico del hospital San Juan de Lurigancho, 2017.

\begin{tabular}{lccc}
\hline & Valor & Df & $\begin{array}{c}\text { Significación asinítítica } \\
\text { (2 caras) }\end{array}$ \\
\hline Chi-cuadrado de Pearson & $1,978^{\circ}$ & 2 &, 372 \\
Razón de verosimilitud & 2,223 & 2 &, 329 \\
Asociación lineal por lineal &, 393 & 1 &, 531 \\
\hline N de casos validos & 20 & \\
\hline a. 4 casillas $(66,7 \%)$ han esperado un recuento menor que 5 . El recuento mínimo esperado es, 35.
\end{tabular}

Respecto al factor tecnológico y el nivel de cumplimiento de registro de lista de chequeo de cirugía segura. Hay más del $20 \%$ de casillas con frecuencias esperadas menores de 5 , por tanto, no se puede usar prueba Chi cuadrado. Se usa la Prueba exacta de Fisher dado que la tabla de contingencia es de $2 \times 2$ y hay dos celdas con frecuencias menores de 5. Si p-value (sig.) $<$ Nivel de significación se rechaza $\mathrm{H} 0$, en caso contrario no se rechaza H0. 0,049 es menor de 0.05 , entonces se rechaza $\mathrm{H} 0$, por tanto, con $5 \%$ de nivel de significación El factor tecnológico y el nivel de cumplimiento de la calidad de registro de lista de chequeo de cirugía segura, no son independientes. (Tabla 4).

Tabla 4. Factor tecnológico y nivel de cumplimiento de registro de lista de chequeo de cirugía segura en el centro quirúrgico del hospital San Juan de Lurigancho, 2017.

\begin{tabular}{|c|c|c|c|c|c|}
\hline & Valor & Df & $\begin{array}{l}\text { Sig. } \\
\text { asinticatica } \\
\text { (2 caras) }\end{array}$ & $\begin{array}{c}\text { Significación } \\
\text { exacta } \\
\text { (2 carras) }\end{array}$ & $\begin{array}{l}\text { Significación } \\
\text { exacta } \\
\text { (1 cara) }\end{array}$ \\
\hline Chi-cuadrado de Pearson & $4,615^{a}$ & 1 &, 032 & & \\
\hline Corrección de continuidad & 2,679 & 1 & 102 & & \\
\hline Razón de verosimilitud & 6,490 & 1 & ,011 & & \\
\hline Prueba exacta de Fisher & & & & ,049 & ,044 \\
\hline Asociación lineal por lineal & 4,385 & 1 & ,036 & & \\
\hline $\mathrm{N}$ de casos válidos & 20 & & & & \\
\hline
\end{tabular}

Según el cumplimiento de los parámetros de la lista de cirugía segura, el $65 \%$ de los encuestados 
cumple con los parámetros de la lista de cirugía segura mientras que el $35 \%$ de encuestados no cumple con dichos parámetros (Tabla 5).

Tabla 5. Cumplimiento de los parámetros de la lista de cirugía segura en el centro quirúrgico del Hospital San Juan de Lurigancho, 2017.

\begin{tabular}{lccc}
\hline & Nivel & $\mathbf{N}^{\mathbf{0}}$ & Porcentaje \\
\hline No cumple & 7 & 35 \\
Cumple & & 13 & 65 \\
\hline & Total & $\mathbf{2 0}$ & $\mathbf{1 0 0 , 0}$ \\
\hline
\end{tabular}

\section{DISCUSIÓN}

De acuerdo a los factores considerados, fueron analizados el factor humano, factor administrativo, factor físico y factor tecnológico. Rivero D, Nolasco A, Puntunet M y Cortés G (9), tomaron en consideración factores similares.

En el estudio se considera que el factor humano no se asocia significativamente al correcto llenado de la lista de verificación de cirugía segura. Según Becerra K (10), las limitaciones existentes para la correcta aplicación de la lista de chequeo son en menor suma la falta de capacitación, responsabilidad y conciencia. Mostrando de esta forma concordancia con lo establecido.

El factor administrativo influye de forma significativa en el llenado de la lista de chequeo de cirugía segura. Figueroa W, Inoñan S (11) identifica la importancia de las actividades administrativas en base a la manifestación del personal correspondiente concluyendo que, oficializar los procesos de capacitación para todo el equipo quirúrgico debe ser un parámetro de carácter obligatorio y no esporádico.

El factor físico no influye de forma significativa en el llenado de la lista de chequeo de cirugía segura. Soria V, André S, Saturno P, Grau M y Carrillo A (12) de forma similar dan a conocer la reducida importancia de los componentes físicos necesarios para el adecuado llenado de la lista de chequeo de cirugía segura.

El factor tecnológico es uno de los principales factores que influyen en el llenado de la lista de chequeo de cirugía segura. Lo cual concuerda con Izquierdo F (13) quien destaca la importancia de los implementos tecnológicos durante el llenado de la lista de chequeo de cirugía segura.

Respecto al cumplimiento en el llenado de la Lista de Chequeo de cirugía segura, este no se realiza de forma adecuada por la complejidad de los factores involucrados. Dicho resultado concuerda con el estudio desarrollado por Rivero
D, Nolasco A, Puntunet M y Cortés G (9) en donde señalan que existen diversos factores que de una $u$ otra forma se relacionan con el cumplimiento en el llenado correcto de la lista de verificación de cirugía segura.

También concuerda con el estudio desarrollado por Pérez R (17), en donde se dan a conocer diversos parámetros que el equipo quirúrgico toma en cuenta en razón de las dificultades específicas para el llenado de las fichas.

\section{CONCLUSIONES}

Se concluye que el factor humano no influye significativamente en el nivel de cumplimiento de registro de lista de chequeo de cirugía segura en el centro quirúrgico. Por otro lado, el factor administrativo influye significativamente el nivel de cumplimiento de registro de lista de chequeo de cirugía segura. Asimismo, el factor físico no influye significativamente en el nivel de cumplimiento de registro de lista de chequeo de cirugía segura. Finalmente, el factor tecnológico influye significativamente en el nivel de cumplimiento de registro de lista de chequeo de cirugía segura. Con respecto al nivel de cumplimiento de registro de lista de chequeo de cirugía segura, este no se desarrolla adecuadamente.

\section{AUTOR DE CORRESPONDENCIA}

Victor Humberto Chero Pacheco

Universidad María Auxiliadora

Av. Canto Bello No 431

Lima 36 - Perú

Teléfono: +51-1-3891212

E-mail:vchp_dent@hotmail.com

\section{REFERENCIAS BIBLIOGRÁFICAS}

1. Organización Mundial de la Salud. La Cirugía Segura Salva Vidas [Internet]. Ginebra: Suiza; 2008 [citado el 20 de marzo 2017]. Disponible en: http://whqlibdoc.who.int/hq/2008/WHO_IE R_PSP_2008.07_spa.pdf

2. Alianza Mundial para la Seguridad del Paciente. Lista OMS de verificación de la seguridad de la cirugía. Manual de aplicación [Internet]. OMS; 2008. [citado el 22 de febrero 2017]. Disponible en: http://whqlibdoc.who.int/hq/2008/WHO_IE R_PSP_2008.05_spa.pdf

3. Ministerio de Salud. Calidad en Salud y Seguridad del Paciente. [citado el 11 de marzo 2017]. Disponible en: 
http://www.minsa.gob.pe/portada/especiales /2010/calidad salud/index.html.

4. Rivero D, Nolasco A, Monserrat L, Cortés G. Nivel de cumplimiento y factores que influyen en la aplicación de la lista de verificación de cirugía segura, Revista Mexicana de enfermería cardiológica. 2012; 20 (2): pp 48.

5. Dackiewicz N, Viteritti L, Marciano B, Bailez B, Merino P, Bortolato D, Jaichenko A, Seminara R, y otros. Lista de verificación de seguridad de la cirugía: logros y dificultades de su implementación en un hospital pediátrico. Arch Argent Pediatr. 2012;110(6): pp. 503.

6. Ministerio de Sanidad y Política Social. Estudio IBEAS. Prevalencia de efectos adversos en hospitales de Latinoamérica [Internet]. 2009 [citado el 16 marzo 2017]. Disponible en: http://www.supersalud.gob.cl/documentacio n/569/articles-7102_recurso_1.pdf.

7. Departamento de enfermería HNAAA. Archivo de producción de centro quirúrgico.2012. Chiclayo. Perú.

8. Revistas Médicas de Colombia. Modelo conceptual y definiciones básicas de la política de seguridad del paciente [Citado el 28 de Marzo 2017]. Disponible en: https://encolombia.com/medicina/revistasmedicas/academedicina/va-

81/respuestadelministerio1/

9. Rivero D, Nolasco A, Puntunet M, Cortés G. Nivel de cumplimiento y factores que influyen en la aplicación de la lista de verificación de cirugía Segura 2012 [Tesis]. México; 2012 [citado el 16 marzo 2017]. Disponible en: http://www.medigraphic.com/pdfs/enfe/en2012/en122b.pdf

10. Becerra K. Experiencias de las enfermeras quirúrgicas respecto a la aplicación de la lista de chequeo de cirugía segura. [Tesis]. Lima Perú; 2012 [citado el 16 marzo 2017]. Disponible en: http://tesis.usat.edu.pe/bitstream/usat/424/1/ TL_Becerra_Eneque_KatherinsMilagros.pd $\mathrm{f}$

11. Figueroa W. Inoñan S. Experiencias de las enfermeras quirúrgicas en el proceso de implementación de la lista de verificación: cirugía segura, hospital iii-1 MINSA, Chiclayo Perú, 2014. [Tesis]. Chiclayo Perú; 2015. [citado el 16 marzo 2017]. Disponible en:

http://publicaciones.usat.edu.pe/index.php/

AccCietna2014/article/view/333/330
12. Soria V; Andre S, Saturno P, Grau M, Carrillo A. Dificultades en la implantación del checklist en los quirófanos de cirugía. [Tesis]. España; 2012 [citado el 16 marzo 2017]. Disponible en: https://www.um.es/calidadsalud/archivos/So ria-Aledo\%20et\%20al.,\%202012.pdf

13. Izquierdo F. Nivel de cumplimiento de la lista de verificación de cirugía segura por el personal del centro quirúrgico del Hospital Nacional Dos de mayo 2015. Lima Perú 2015 [citado el 16 marzo 2017]. Disponible en:

http://cybertesis.unmsm.edu.pe/xmlui/handl e/cybertesis $/ 5078$

14. Pérez R. Percepción del equipo quirúrgico sobre la utilidad y aplicación de la lista de verificación para una cirugía cardiovascular segura en el servicio de centro quirúrgico del Instituto Nacional Cardiovascular de EsSalud - 2014. [Tesis]. Lima Perú; 2015 [citado el 16 marzo 2017]. Disponible en: $\mathrm{http} / / /$ ateneo.unmsm.edu.pe/ateneo/bitstrea m/123456789/5021/1/Perez_Quispe_Rocio_ Marcely_2015.pdf 